\title{
Efficacy of a Topical Gelatin-Thrombin Matrix Sealant in Reducing Postoperative Drainage Following Anterior Cervical Discectomy and Fusion
}

\author{
Quan You Li, Osung Lee, Ho Sung Han, Gang-Un Kim, Chee Kean Lee, \\ Sung Shik Kang, Myung Ho Lee, Hyeon Guk Cho, Ho-Joong Kim, Jin S. Yeom \\ Spine Center and Department of Orthopaedic Surgery, Seoul National University Bundang Hospital, \\ Seoul National University College of Medicine, Seongnam, Korea
}

Study Design: Retrospective fusion level(s)-, age-, and gender-matched analysis.

Purpose: To determine whether the application of a topical gelatin-thrombin matrix sealant (Floseal) at the end of anterior cervical discectomy and fusion (ACDF) can reduce the amount of postoperative hemorrhage.

Overview of Literature: The effect of the matrix sealant in decreasing postoperative hemorrhage following ACDF has not been reported.

Methods: Matrix sealant was ( $n=116$, study group) or was not applied ( $n=58$, control group) at the end of ACDF. Patients were selected by 1:2 matching criteria of fusion level(s), age, and gender. Seven parameters described below were compared between the two groups.

Results: The total drain amount for the first 24 hours $(8 \pm 9$ versus $27 \pm 22 \mathrm{~mL})$, total drain amount until the 8-hour drainage decreased to $\leq 10 \mathrm{~mL}(8 \pm 10$ versus $33 \pm 26 \mathrm{~mL})$, and the total drain amount until 6 AM on the first postoperative day $(7 \pm 8$ versus $24 \pm 20 \mathrm{~mL})$ were significantly lower in the study group than the control group (all $p<0.001$ ). The time for the 8-hour drainage to decrease to $\leq 10 \mathrm{~mL}$ was significantly lower in the study group ( $10 \pm 5$ versus $26 \pm 14$ hours, $p<0.001)$. The 8 -hour drainage decreased to $\leq 10 \mathrm{~mL}$ on the operation day in most patients (88\%) in the study group versus mostly on the first $(48 \%)$ or second $(33 \%)$ postoperative day in the control group $(p<0.001)$. The total drain amount until 6 AM on the first postoperative day was $0 \mathrm{~mL}$ in $43 \%$ of patients in the study group and in $7 \%$ in the control group $(p<0.001)$. No patient in either group required hematoma evacuation.

Conclusions: Application of the topical matrix sealant at the end of ACDF can significantly reduce the amount of postoperative hemorrhage.

Keywords: Cervical vertebrae; Hemostasis; Postoperative hemorrhage; Floseal matrix; Spinal fusion

\section{Introduction}

One of the most catastrophic complications of anterior cervical discectomy and fusion $(\mathrm{ACDF})$ is postoperative hemorrhage, which may result in dyspnea, respiratory arrest, and death [1-6]. Surgeons need to be alert for this complication and try their best to prevent it [7-11]. One of the various methods to minimize postoperative hemor-

\footnotetext{
Received Nov 3, 2015; Accepted Nov 13, 2015

Corresponding author: Jin S. Yeom

Spine Center and Department of Orthopaedic Surgery, Seoul National University Bundang Hospital, Seoul National University College of Medicine, 166 Gumi-ro, Bundang-gu, Seongnam 13620, Korea

Tel: +82-31-787-7195, Fax: +82-31-787-4056, E-mail: highcervical@gmail.com
} 
rhage is the local application of a hemostatic agent. Even though a topical gelatin-thrombin matrix sealant (Floseal; Baxter Healthcare Corp., Westlake Village, CA, USA) has been used for hemostasis in various operations [12-14], its effect in decreasing postoperative hemorrhage following ACDF has not been reported as far as we know. In addition, we could not find a report on the efficacy of its use at the end of surgery instead of during surgery.

This study aimed to determine whether the application of a topical gelatin-thrombin matrix sealant at the end of the surgery decreased the amount of postoperative hemorrhage. The amount of the postoperative drainage for patients in whom the matrix sealant was applied at the end of surgery (study group) was compared with those for whom the sealant was not applied (control group) in a retrospective fusion level(s)-, age-, and gender-matched analysis.

\section{Materials and Methods}

\section{Study design and participants}

This study was approved by the Institutional Review Board of the Seoul National University Bundang Hospital. Surgeries performed from August 2007 to December 2008 did not involve the use of the gelatin-thrombin matrix sealant, while surgeries performed from February 2009 to February 2015 involved the topical application of the gelatin-thrombin matrix sealant at the conclusion of surgery in an effort to reduce postoperative hemorrhage. The initial inclusion criteria for both groups were degenerative cervical disorders including spondylosis and disc herniation, or ossification of posterior longitudinal ligaments; ACDF with plate fixation at the vertebral level(s) between $\mathrm{C} 3$ and $\mathrm{T} 1$; and age $\geq 20$ years. The initial exclusion criteria were: infection, trauma, or tumors; additional cervical operation(s); anticoagulation therapy not discontinued until 1 week prior to the index surgery; inherited or acquired bleeding disorders; any unclear or defective clinical data; and unintended accidental removal of the suction drain.

For the control group, we initially enrolled all patients fulfilling the above selection criteria from those patients in the last author's cervical spine surgery registry. For each patient in the control group, two patients were selected for the study group, with the matching criteria of fusion level(s), age (within \pm 5 years), and gender. For example, for a 62.4-year old woman who underwent C5C6-C7 ACDF in the control group, two women who were between 57.4- and 67.4-years-of-age and underwent C5C6-C7 ACDF were selected for the study group. When there were three or more patients for the study group who fulfilled the matching criteria for a patient in the control group, only two patients whose operation dates were closer to that of the matched study group patient were selected. When there was no or only one patient for the study group who fulfilled the matching criteria for a patient in the control group, all of the patients were excluded.

\section{Surgical procedures, application of the hemostatic agent, and postoperative measurement of drainage}

All operations were carried out by the last author. In the study group, hemostasis was performed during the surgical approach and decompression using bipolar electrocautery or a thrombin-soaked gelatin sponge (Spongostan, Ethicon, Sommerville, NJ, USA) and cottonoid pledgets. The gelatin-thrombin matrix sealant was not used during these procedures. After decompression was completed (Fig. 1A), a small amount $(<1 \mathrm{~mL})$ of the gelatin-thrombin matrix sealant was applied in the disc space (Fig. 1B) and compression was applied using a cottonoid pledget for a few tens of seconds (Fig. 1C), which resulted in satisfactory control of hemorrhage from the epidural venous plexi and other areas in the disc space. Excess amount of the matrix sealant was removed. During the subsequent placement of a bone graft or cage and plate fixation, the matrix sealant was not used. After all of these procedures were completed (Fig. 2A), all of the remaining matrix sealant was applied around the plate and any sources of bleeding (Fig. 2B). In all patients, only a single $5 \mathrm{~mL}$ kit of matrix sealant was used. Cottonoid pledgets (Fig. 2C) were placed over the matrix sealant and a $10 \times 10 \mathrm{~cm}$ gauze pad was added. After inserting and tagging a suction drain and taking intraoperative radiographs, the gauze and cottonoid pledgets were removed. The matrix sealant was not removed at this time. The wound was closed in layers and negative pressure suction was applied to the drain after wound closure. In the control group, the same surgical procedures were done, with the exception of the omission of the matrix sealant.

Postoperatively, drainage was measured every 8 hour in both groups. The drain was usually removed when the drainage for the most recent 8 hours was $<10 \mathrm{~mL}$. 

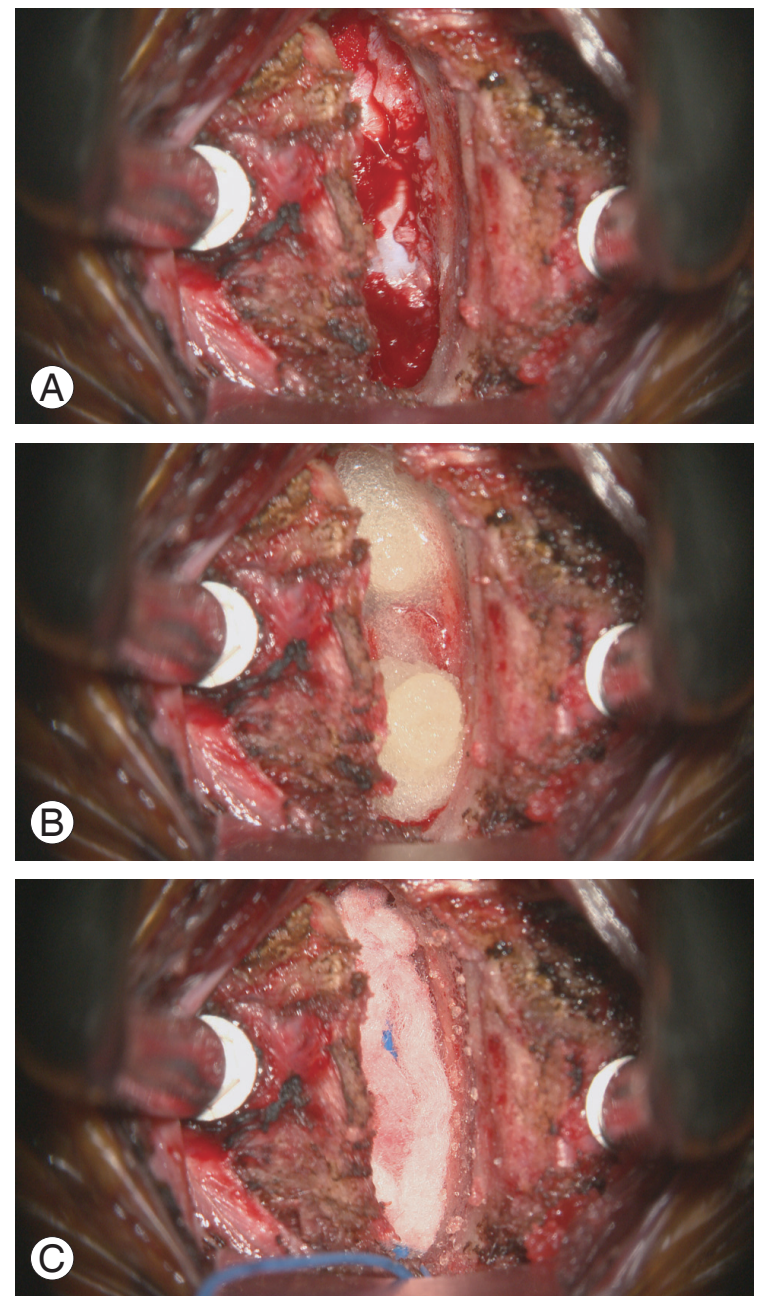

Fig. 1. Intraoperative photographs taken after completion of decompression (A), application of the matrix sealant in the disc space (B), and placement of a cottonoid pledget (C) are shown.

\section{Data collection and analyses}

Four independent orthopaedic surgeons who had not been involved in the care of the enrolled patients conducted patient selection, and data collection and analyses. All demographic and clinical data including the volume of drainage were obtained from the cervical spine surgery registry and electronic medical records.

We compared seven parameters between the two groups. First, total drain amount $(\mathrm{mL})$ for the first 24 hours after surgery was compared. Second, the time interval (hours) from when surgery (wound closure) was completed to when the 8-hour drainage decreased to $\leq 10 \mathrm{~mL}$ was compared. Third, the number of postoperative day(s) when the 8-hour drainage decreased to $\leq 10 \mathrm{~mL}$ was com-
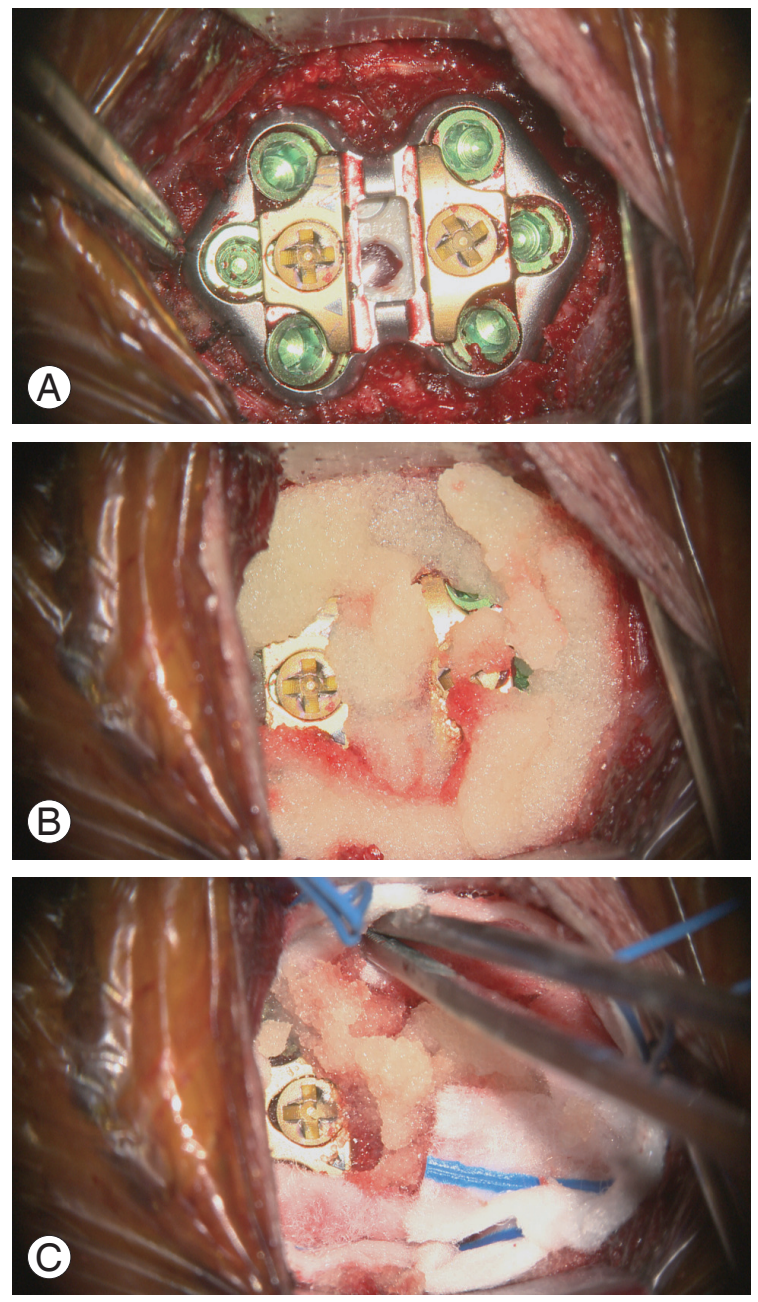

Fig. 2. Intraoperative photographs taken after plate fixation (A), application of the matrix sealant around the plate (B), and placement of cottonoid pledgets (C) are shown.

pared. Fourth, the total drain amount until the 8-hour drainage decreased to $\leq 10 \mathrm{~mL}$ was compared. Fifth, the total drain amount until $6 \mathrm{AM}$ on the first postoperative day was compared. Sixth, the number of patients in whom the total drain amount until $6 \mathrm{AM}$ was $0 \mathrm{~mL}$ on the first postoperative day was compared. Seventh, the number of patients who required hematoma evacuation for the treatment of either respiratory problem caused by retropharyngeal hematoma or neurologic deficit caused by epidural hematoma was compared.

Student's $t$-test was used for continuous variables and the chi-square test was used for comparison of proportions. Statistical analyses were carried out with MedCalc statistical software ver. 15.8 (MedCalc Software, Ostend, Belgium). The level of significance was set at a 2 -tailed $p<0.05$. 
Table 1. Demographic and basic clinical data

\begin{tabular}{|c|c|c|c|}
\hline \multirow{2}{*}{ Variable } & \multicolumn{2}{|c|}{ Group } & \multirow{2}{*}{$p$-value } \\
\hline & Control $(\mathrm{n}=58)$ & Study $(n=116)$ & \\
\hline Age (yr) & $53 \pm 12$ & $52 \pm 11$ & $0.94^{\text {a) }}$ \\
\hline Gender & & & $>0.99^{\text {b) }}$ \\
\hline Male & $41(71)$ & $82(71)$ & \\
\hline Female & $17(29)$ & $34(29)$ & \\
\hline Fusion level(s) & & & $>0.99^{\text {b) }}$ \\
\hline C3-C4 & $4(7)$ & $8(7)$ & \\
\hline C4-C5 & $7(12)$ & $14(12)$ & \\
\hline C5-C6 & $20(34)$ & $40(34)$ & \\
\hline C6-C7 & $16(28)$ & $32(28)$ & \\
\hline C4-C5-C6 & $2(3)$ & $4(3)$ & \\
\hline C5-C6-C7 & $8(14)$ & $16(14)$ & \\
\hline C6-C7-T1 & $1(2)$ & $2(2)$ & \\
\hline Disorders & & & $0.43^{b)}$ \\
\hline Cervical spondylosis & $38(66)$ & $81(70)$ & \\
\hline Soft disc herniation & $17(29)$ & $25(22)$ & \\
\hline Ossification of posterior longitudinal ligament & $3(5)$ & $10(9)$ & \\
\hline Time to 6 AM on the first postoperative day (hr) & $15.2 \pm 2.8$ & $15.4 \pm 2.9$ & $0.71^{\mathrm{a})}$ \\
\hline
\end{tabular}

Values are presented as mean \pm standard deviation or number (\%).

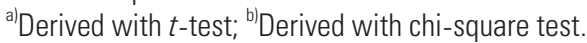

\section{Results}

\section{Demographic and basic clinical data}

Sixty seven patients identified from the cervical spine surgery registry fulfilled the initial inclusion/exclusion criteria for the control group. During the 1:2 matching, 9 patients were excluded because they did not have two matched study group patients. Therefore, 58 patients were finally enrolled for the control group. For the study group, among the 349 patients fulfilling the initial inclusion/exclusion criteria, 116 patients were finally selected after the 1:2 matching.

Table 1 summarizes demographic and basic clinical data of both groups. Age, gender, and number of fusion level(s) did not differ significantly between the two groups, as expected given the matching criteria design ( $p=0.94,>0.99$, and $>0.99$, respectively). The most common disorders were cervical spondylosis with myelopathy and/or radiculopathy in both groups, followed by soft disc herniation and ossification of the posterior longitudinal ligament. There was no statistically significant difference between the two groups $(p=0.43)$. The time from the completion of surgery to $6 \mathrm{AM}$ on the first postoperative day was not significantly different between the two groups $(p=0.71)$.

\section{Drainage amount and hematoma evacuation}

The results are summarized in Table 2 . The total drain amount for the first 24 hours after surgery was significantly lower in the study group than in the control group $(8 \pm 9$ vs. $27 \pm 22 \mathrm{~mL} ; p<0.001)$. The time for the 8 -hour drainage to decrease to $\leq 10 \mathrm{~mL}$ was significantly lower in the study group than in the control group ( $10 \pm 5$ vs. $26 \pm 14$ hours; $p<0.001)$. The 8 -hour drainage decreased to $\leq 10$ $\mathrm{mL}$ on the day of operation in most study group patients $(102 / 116,88 \%)$, while it did on the first $(28 / 58,48 \%)$ or second $(19 / 58,33 \%)$ postoperative day in the majority of patients in the control group. The difference was statistically significant $(p<0.001)$. Total drain amount until the 8 -hour drainage decreased to $\leq 10 \mathrm{~mL}$ was significantly 
Table 2. Seven analyzed parameters

\begin{tabular}{|c|c|c|c|}
\hline \multirow{2}{*}{ Parameter } & \multicolumn{2}{|c|}{ Group } & \multirow{2}{*}{$p$-value } \\
\hline & Control $(n=58)$ & Study $(n=116)$ & \\
\hline Total drain amount for the first 24 hours (mL) & $27 \pm 22$ & $8 \pm 9$ & $<0.001^{\text {a) }}$ \\
\hline Time to 8-hour drainage $\leq 10 \mathrm{~mL}(\mathrm{hr})$ & $26 \pm 14$ & $10 \pm 5$ & $<0.001^{\text {a) }}$ \\
\hline Number of patients with 8-hour drainage decreased to $\leq 10 \mathrm{~mL}$ & & & $<0.001^{\text {b) }}$ \\
\hline On the operation day & $11(19)$ & $102(88)$ & \\
\hline On the postoperative 1st day & $28(48)$ & $13(11)$ & \\
\hline On the postoperative 2 nd day & $19(33)$ & $1(1)$ & \\
\hline Total drain amount until 8-hour drainage $\leq 10 \mathrm{~mL}(\mathrm{~mL})$ & $33 \pm 26$ & $8 \pm 10$ & $<0.001^{\text {a) }}$ \\
\hline Total drain amount until 6 AM on POD 1 (mL) & $24 \pm 20$ & $7 \pm 8$ & $<0.001^{\mathrm{a})}$ \\
\hline Number of patients with total drain amount until 6 AM on POD 1 & & & $<0.001^{\text {b) }}$ \\
\hline$=0 \mathrm{~mL}$ & $4(7)$ & $50(43)$ & \\
\hline$>0 \mathrm{~mL}$ & $54(93)$ & $66(57)$ & \\
\hline Hematoma evacuation & & & - \\
\hline Required & 0 & 0 & \\
\hline Not required & $58(100)$ & $116(100)$ & \\
\hline
\end{tabular}

Values are presented as mean \pm standard deviation or number (\%).

POD, postoperative day.

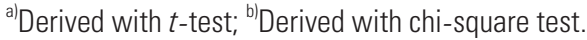

lower in the study group than in the control group $(8 \pm 10$ vs. $33 \pm 26 \mathrm{~mL} ; p<0.001)$. Total drain amount until $6 \mathrm{AM}$ on the first postoperative day was significantly lower in the study group than in the control group ( $7 \pm 8$ vs. $24 \pm 20$ $\mathrm{mL} ; p<0.001)$. Total drain amount until $6 \mathrm{AM}$ on the first postoperative day was $0 \mathrm{~mL}$ in 50 of $116(43 \%)$ patients in the study group and 4 of 58 (7\%) patients in the control group $(p<0.001)$. No patient in either group required hematoma evacuation.

\section{Discussion}

Postoperative hemorrhage following ACDF can result in disastrous complications [1-6]. Retropharyngeal hematoma may lead to airway obstruction and respiratory arrest, and epidural hematoma may cause neurologic complications. Minimizing these complications necessitates meticulous hemostasis and reduction in postoperative hemorrhage. Topical hemostatic agents may be used for this purpose. One of the most commonly used agents in various surgical fields is gelatin-thrombin matrix sealant [12-14]. Despite its popularity, we could not find a report on its effect in reducing the amount of postopera- tive hemorrhage following ACDF, which prompted the present retrospective matched pair analysis. While most surgeons use gelatin-thrombin matrix sealant during the main operative procedures, we used it only at the end of surgery to minimize postoperative hemorrhage instead of intraoperative hemorrhage, considering the cost of the matrix sealant.

\section{Selection of parameters for analyses}

As indices of the amount of postoperative hemorrhage, six parameters were selected (Table 2). In determining these parameters, several commonly used ones were excluded. First, we did not measure or compare the drain removal time and the total volume of drainage before drain removal, because the operating surgeon (the last author) did not strictly follow the protocol for drain removal (8-hour drainage $\leq 10 \mathrm{~mL}$ ) in all cases. He tended to maintain the drain for a longer time in the past (i.e., in the control group). Therefore, comparison of those two parameters should have resulted in favorable results for the study group. For this reason, we did not include those parameters in our analyses for the sake of a fair compari- 
son. Second, we did not compare the length of hospital stay. Most patients refuse to be discharged for several days after surgery in this country, particularly because of the very low cost for admission. The hospital cost for one day is $<50$ US dollars in this country, where the medical cost is determined and strictly controlled by the government. Therefore, drain output has nothing to do with length of stay in this country. For this reason, we did not analyze the length of stay.

On the other hand, we included the total drain amount until $6 \mathrm{AM}$ on the first postoperative day and the number of patients in whom the total drain amount until $6 \mathrm{AM}$ was $0 \mathrm{~mL}$ on the first postoperative day. Although the time from the completion of surgery to 6 AM differed from patient-to-patient, we included these two parameters for two reasons. First, the first night following surgery is regarded by surgeons as the more dangerous period because of the potentially life-threatening complications that can result from postoperative hemorrhage. Secondly, many surgeons check the amount of drainage during surgical rounds the morning following surgery and determine at this time whether to remove the drain or not. The time interval from the completion of surgery to $6 \mathrm{AM}$ was not significantly different between the two groups $(p=0.71$ ) (Table 1). Therefore, comparison of these two parameters was not inappropriate. Of note, the amount of drainage was measured based on the quantity in the drainage bag. Blood or discharge in the drainage tube was not measured in order to maintain the sterility of the postoperative wound. Therefore, when the blood or discharge was draining only along the tube and not in the bag, it was considered as $0 \mathrm{~mL}$. Both the control and study groups were measured using the same methodology.

\section{Data interpretation}

The present data demonstrate that use of the gelatinthrombin matrix at the end of the ACDF can effectively reduce postoperative hemorrhage. All of the six parameters related with postoperative drain output, including the total drain amount for the first 24 hours after surgery, time from wound clousure to when the 8-hour drainage decreased to $\leq 10 \mathrm{~mL}$ and the total drain amount until this time, and the total drain amount until $6 \mathrm{AM}$ on the first postoperative day, were significantly lower in the study group than in the control group. In addition, the $p$-values were sufficiently low $(p<0.001)$ for all of the six param- eters. This provides sound evidence that the application of gelatin-thrombin matrix after completion of the main procedures of ACDF is efficacious in decreasing postoperative hemorrhage. However, this study did not prove the efficacy of the gelatin-thrombin matrix in preventing the occurrence of postoperative airway obstruction caused by retropharyngeal hematoma or neurologic deficit caused by epidural hematoma, because there were no such complications in either groups. These are very rare complications and the sample size of this study might not have been large enough for such analysis. A much larger scale study is required for this analysis.

\section{Limitations}

This study has several limitations. It is not a prospective randomized study. Because the principal author was convinced of the positive effect of the gelatin-thrombin matrix sealant, he did not conduct a prospective study. This study is, however, a fusion level(s)-, age-, and gendermatched analysis, and the matching criteria was strictly kept during patient selection. In addition, all the operations were carried out by a single surgeon in a single institution. However, the influence of potential confounders cannot be definitely excluded in a retrospective study. For example, the operating surgeon's skill likely improved with time, favoring the study group. On the other hand, the surgeon tended to carry out more aggressive procedures, such as total uncoforaminotomy, resection of posterior longitudinal ligament, and partial corpectomy with time, and yielded more procedures to fellows and residents under his supervision with time, favoring the control group. A second limitation is the lack of patients with long-level fusion. Because the operating surgeon preferred selective surgery for anterior cases and tended to choose posterior or anteroposterior surgery for long level cases, we could not find patients fulfilling our strict 1:2 matching criteria among those patients who underwent multi-level ACDF. However, because gelatin-thrombin matrix sealant showed definite positive effect even in 1- or 2-level ACDF cases, we believe it will have more pronounced effect in multi-level ACDF. Third, even though this study demonstrated that postoperative hemorrhage can be sufficiently reduced by using the matrix sealant, it did not prove its clinical benefit in prevention of related complications because there were no complications related with postoperative hemorrhage in either group. However, the total 
drain amount until $6 \mathrm{AM}$ on the first postoperative day (15.4 \pm 2.9 hours after surgery) was significantly reduced to $7 \pm 8 \mathrm{~mL}$ with the use of the matrix sealant and $43 \%$ of those patients had no drainage. The data should inspire confidence in surgeons of the outcome in the first night following surgery concerning catastrophic hemorrhagerelated complications. Still, more scientific evidences concerning about this issue are needed. We leave this decision to the readers of this paper. Fourth, we assumed that the amount of postoperative drainage reflect the amount of postoperative hemorrhage without any validation. Fifth, as this retrospective study was designed to look for the efficacy of the matrix sealant in reducing postoperative hemorrhage, its side effects were not particularly assessed. However, to our best awareness, no patient developed side effects of this sealant.

\section{Conclusions}

This retrospective fusion level(s)-, age-, and gendermatched analysis demonstrates that application of a topical gelatin-thrombin matrix sealant at the end of ACDF can significantly reduce the amount of postoperative hemorrhage.

\section{Conflict of Interest}

No potential conflict of interest relevant to this article was reported.

\section{Acknowledgments}

We thank and acknowledge Ms. Young Hee Ahn, a physician assistant at our institution; and Ms. Junga Park, Song Yi Kim, Chae Won Park, spine research coordinators at our institution, for their contributions to the project.

\section{References}

1. Sagi HC, Beutler W, Carroll E, Connolly PJ. Airway complications associated with surgery on the anterior cervical spine. Spine (Phila Pa 1976) 2002;27:949-53.

2. Palumbo MA, Aidlen JP, Daniels AH, Thakur NA, Caiati J. Airway compromise due to wound hematoma following anterior cervical spine surgery. Open Orthop J 2012;6:108-13.

3. Dagli M, Er U, Simsek S, Bavbek M. Late results of anterior cervical discectomy and fusion with interbody cages. Asian Spine J 2013;7:34-8.

4. Pallud J, Belaid H, Aldea S. Successfull management of a life threatening cerebellar haemorrhage following spine surgery: a case report. Asian Spine J 2009;3:32-4.

5. Song KJ, Choi BY. Current concepts of anterior cervical discectomy and fusion: a review of literature. Asian Spine J 2014;8:531-9.

6. Buerba RA, Giles E, Webb ML, Fu MC, Gvozdyev B, Grauer JN. Increased risk of complications after anterior cervical discectomy and fusion in the elderly: an analysis of 6253 patients in the American College of Surgeons National Surgical Quality Improvement Program database. Spine (Phila Pa 1976) 2014;39:2062-9.

7. Yeom JS, Buchowski JM, Shen HX, Liu G, Bunmaprasert T, Riew KD. Effect of fibrin sealant on drain output and duration of hospitalization after multilevel anterior cervical fusion: a retrospective matched pair analysis. Spine (Phila Pa 1976) 2008;33: E543-7.

8. Cho SK, Yi JS, Park MS, et al. Hemostatic techniques reduce hospital stay following multilevel posterior cervical spine surgery. J Bone Joint Surg Am 2012;94: 1952-8.

9. Tsutsumimoto T, Shimogata M, Ohta H, Yui M, Yoda I, Misawa H. Tranexamic acid reduces perioperative blood loss in cervical laminoplasty: a prospective randomized study. Spine (Phila Pa 1976) 2011;36:1913-8.

10. Schubert M, Merk S. Retrospective evaluation of efficiency and safety of an anterior percutaneous approach for cervical discectomy. Asian Spine J 2014;8: 412-20.

11. Wada K, Murata Y, Kato Y. Surgical outcome for hemodialysis-related upper cervical lesions. Asian Spine J 2015;9:699-704.

12. Oz MC, Cosgrove DM 3rd, Badduke BR, et al. Controlled clinical trial of a novel hemostatic agent in cardiac surgery: the Fusion Matrix Study Group. Ann Thorac Surg 2000;69:1376-82.

13. Krishnan S, Conner TM, Leslie R, Stemkowski S, Shander A. Choice of hemostatic agent and hospital length of stay in cardiovascular surgery. Semin Cardiothorac Vasc Anesth 2009;13:225-30.

14. Gazzeri R, Galarza M, Alfier A. Safety biocompatibility of gelatin hemostatic matrix (Floseal and Surgiflo) in neurosurgical procedures. Surg Technol Int 2012; 22:49-54. 